\title{
Dual protection to address the global syndemic of HIV and unintended pregnancy in Brazil
}

\author{
Kiyomi Tsuyuki, ${ }^{1}$ Jessica D Gipson, ${ }^{2}$ Lianne A Urada, ${ }^{3}$ \\ Regina Maria Barbosa, ${ }^{4}$ Donald E Morisky ${ }^{5}$
}

\begin{abstract}
${ }^{1}$ NIDA Post-doctoral Fellow, Division of Global Public Health, Department of Medicine, University of California, San Diego, La Jolla, CA, USA ${ }^{2}$ Assistant Professor, Department of Community Health Sciences, Fielding School of Public Health, University of California, Los Angeles (UCLA), USA

${ }^{3}$ Assistant Professor, Division of Global Public Health, Department of Medicine, University of California, San Diego, La Jolla, CA, USA ${ }^{4}$ Professor, Population Studies Center (NEPO), University of Campinas (UNICAMP), Campinas, Brazil

${ }^{5}$ Professor, Department of Community Health Sciences, Fielding School of Public Health, University of California, Los Angeles (UCLA), USA
\end{abstract}

\section{Correspondence to} Dr Kiyomi Tsuyuki, Division of Global Public Health, Department of Medicine, University of California, 9500 Gilman Drive, MC 0507, San Diego, La Jolla, CA 920930507, USA; ktsuyuki@gmail.com

Received 9 January 2015 Revised 29 September 2015 Accepted 23 November 2015 Published Online First

15 February 2016

CrossMark

To cite: Tsuyuki $K$, Gipson JD, Urada LA, et al. J Fam Plann Reprod Health Care 2016;42:271-279.

\begin{abstract}
Background Syndemic HIV and unintended pregnancy is prevalent in Brazil, where $79 \%$ of female HIV cases occur in women of reproductive age and $55 \%$ of all pregnancies are unintended. Although increasing condom use to prevent HIV may decrease non-barrier contraception and increase unintended pregnancy, few studies focus on dual protection or dual methods (condoms with another modern contraceptive)
\end{abstract}

Aim To describe the correlates of dual method use and consistent condom use in women of reproductive age in Brazil.

Method Data are from the 2006 Pesquisa Nacional de Demografia e Saúde da Mulher e da Criança, a decennial nationally representative household survey of women of reproductive age in Brazil. Multivariate logistic regression models identify the socio-demographic, sexual debut, fertility and relationship factors associated with dual method use and consistent condom use.

Results Two-thirds of contracepting women in Brazil used dual protection (40\% exclusive condoms, 27\% dual methods). Consistent condom use in the past year occurred among $61 \%$ of exclusive condom users and $27 \%$ of dual method users. Dual methods (vs exclusive condoms) was associated with some high school education [relative risk ratio $(R R R)=1.69, p<0.05$ ], living in the Southern region $(R R R=1.59, p<0.01)$, and number of children ( $R R R=1.22, p<0.01)$, net of other factors. Consistent condom use was associated with condom use at sexual debut [adjusted odds ratio $(A O R)=1.84, p<0.001$ ], wants no (more) children ( $A O R=1.86, p<0.001)$, single/separated relationship status ( $A O R=2.77 /$ 2.45, $p<0.001$ ) and using exclusive condoms (vs dual methods: $A O R=0.19, p<0.001$ ).

Conclusions Findings highlight that targeting and delivering integrated HIV and family planning services should focus on completed/ large families. single/separated individuals, and promoting dual protection at sexual debut.

\section{Key message points}

- In a Brazilian national sample of contracepting women (aged 15-49 years), $27 \%$ used dual methods to prevent HIV/sexually transmitted infections (STIs) and pregnancy. Forty-seven per cent reported consistent condom use.

- Dual methods associated with more children and inconsistent condom use. Consistent condoms associated with condoms at sexual debut, not wanting more children, and being single/ separated.

- Syndemic HIV/STI and family planning services may usefully target completed/ large families (wanting no more children), single/separated individuals, non-exclusive relationships, and condoms at sexual debut.

\section{INTRODUCTION}

Gender-based inequities, lack of health care access, and biological vulnerability increases women's risk of HIV infection and unintended pregnancy. ${ }^{1} \mathrm{HIV}$ is the leading cause of death for reproductiveaged women worldwide, ${ }^{1}$ followed in close second by maternal death. ${ }^{2}$ Women make up more than half the 35 million people living with HIV, most contracting HIV heterosexually. ${ }^{1}$ Women also have two to eight times the odds of contracting HIV from men during sex than vice versa. ${ }^{3}$ Moreover, $40 \%$ of all pregnancies are unintended (unwanted/mistimed), ${ }^{4}$ highlighting opportunities for improved contraception.

Brazil contends with syndemic HIV and unintended pregnancy. Women make up 
a sizeable demographic of those living with HIV $(n=265251,1980-2014) .^{5}$ The overall HIV rate among women has stabilised, but continues to grow in young women (15-19 years), elderly women (55+ years) and certain regions (South, North, Northeast). ${ }^{5}$ Since the 1990s, the Brazilian AIDS Program and Ministry of Health have supported HIV prevention and condom use campaigns, school-based sex education and condom literacy programmes, and the national distribution of free condoms. ${ }^{6}$ Nevertheless, $79 \%$ of new female HIV cases occur in reproductive-aged women, ${ }^{5}$ and most recent national data indicate that only $57 \%$ report past year condom use. $^{7}$

Unintended pregnancy is also a significant problem in Brazil. National estimates report that $25 \%$ of all births are mistimed and $30 \%$ are unwanted. ${ }^{8}$ Unintended pregnancy disproportionately affects women with low socioeconomic status ${ }^{7-15}$ and women in certain regions (South, Northeast). ${ }^{10} 12$ Unintended pregnancy is exacerbated as a public health issue because women do not have access to safe/legal abortion. ${ }^{16}$ In the 2010 national survey, 17$22 \%$ of reproductive-aged women reported a lifetime prevalence of abortion, ${ }^{17}$ with consequences from unsafe abortion the main cause of maternal death in Brazil. ${ }^{18}{ }^{19}$ High prevalence of unintended pregnancy and abortion reflects an unmet need for family planning, which Brazil has struggled to provide through its universal health care system. ${ }^{14} 1520$

Syndemic HIV and unintended pregnancy calls for integrated prevention efforts as the use of condoms and non-barrier contraceptive methods affect each other. ${ }^{21-23}$ Dual protection is the simultaneous protection against sexually transmitted infections (STIs, including HIV) and unintended pregnancy ${ }^{24}$ and is achieved by the use of a single method (condoms) or dual methods (condoms with another contraceptive). Although condoms can be highly effective in preventing pregnancy and disease transmission with perfect clinical use ${ }^{25}$ the typical contraceptive failure of male condoms $(18 \%)$ is much higher than that of other methods, like the birth control pill (9\%), hormonal injection (6\%), and the intrauterine device (0.2$0.8 \%){ }^{26}$ Although still uncertain, some evidence indicates that promoting condom use for HIV/STIs prevention may result in the declining use of nonbarrier contraception and increasing unintended pregnancy and abortion. ${ }^{27}$

Few studies focus on dual protection or dual methods. Although condoms are the mainstay of dual protection, ${ }^{24}$ dual method use is posited as the most effective way to achieve dual protection ${ }^{28}$ especially for women who want fertility control but cannot consistently negotiate condom use. Dual method use can help to reduce unintended pregnancy and prevent mother-to-child HIV transmission as effectively as antiretroviral treatment. ${ }^{29}{ }^{30}$ However, more research is needed to understand how decisions to use condoms and dual methods are affected by factors like fertility desires and relationship status. For example, as relationships become more serious, partners tend to transition from condoms to non-barrier contraception, ${ }^{22}$ with dual method users reporting more inconsistent condom use than exclusive condom users. $^{31-33}$

To our knowledge, there has been no national study in Brazil that examines dual protection and dual methods in fecund women of reproductive age. Most studies that investigate this are in samples of women living with $\mathrm{HIV}^{34}{ }^{35}$ or adolescents ${ }^{36-38}$ and regionally focus on sub-Saharan Africa ${ }^{34} 39$ or developed countries, ${ }^{36-38}$ with some considering adult women in the general population ${ }^{39} 40$ but none in the Latin American region. Nevertheless, several studies find that Brazilians acknowledge the dual protection provided by condoms. For example, in a nationally representative study of reproductive-aged women, 90\% report that condoms protect against both pregnancy and STIs. ${ }^{7}$ In a qualitative study in the Northeast, Brazilian youth cite that condoms offer dual protection. $^{41}$ In another study, sterilised women who acknowledged that condoms protect against STIs reported condom use, whereas those who only acknowledged condoms as contraception did not report use. ${ }^{42}$

This article describes the correlates of dual method use and consistent condom use among fecund Brazilian women who use modern contraception. First, we describe the sociodemographics, fertility desires, and relationship status of women by method used. Second, we identify factors associated with dual method use. Third, we identify factors associated with consistent condom use among women who report both forms of dual protection. Findings from this article identify characteristics that promote dual protection and suggest areas for targeted intervention to promote dual method use and consistent condom use.

\section{METHODS}

\section{Data}

This study reports findings from a secondary data analysis of the most recent Brazilian national survey, Pesquisa Nacional Demografia e Saúde da Criança e da Mulher (PNDS-2006). ${ }^{43}$ The decennial PNDS, modelled on the Demographic Health Surveys, utilises a two-stage stratified sampling design and is representative of reproductive-aged women who live in private households (including favelas). ${ }^{43}$ In the first sampling stage, 10 strata were composed to include rural and urban areas of the five Brazilian macro-regions. Using sectors established by the 2000 census, 1088 census sectors were randomly selected using simple random cluster sampling. A total of 760 urban and 328 rural PSUs were selected. In the second sampling stage, 12 households were randomly selected per sector. When 
the household did not have an eligible woman (aged $15-49$ years) present, it was substituted with an eligible household closest on the list. A total of 14617 households were chosen, yielding 17456 eligible women, and 15575 women were interviewed (89\% response rate). ${ }^{43}$

Sample weights included household weights to adjust for non-responsiveness in sectors, accounting for the presence of more than one woman in a household, and adjusting for absence/refusal to participate. ${ }^{43}$ Grand sample weights were calibrated (based on the PNAD $2006^{44}$ ) using key variables, including sample strata (region, rural/urban), sex, age distribution, total number of households with at least one woman aged $15-49$ years old, and total number of households. ${ }^{45}$ The PNDS final report details information. ${ }^{7}$ Procedures for data collection were approved by the institutional review boards of Macro Institutional Inc., Sexually Transmitted Diseases/AIDS Reference and Training Center of Sao Paulo's Health Secretariat, and secondary analysis was approved by University of California, Los Angeles (IRB\#11-002445).

\section{Sample}

This study analyses fecund women who report using modern contraception and have complete data $(n=6017)$. We also examine consistent condom use among a sub-sample of condom users $(n=3961)$. We excluded women who used no method $(n=758 ; 11 \%)$ or traditional methods $(n=223 ; 3 \%)$; they were older, less formally educated, had more children, were less likely to use condoms/contraception at sexual debut, and were more likely to be married than included women.

\section{Measures}

Dual protection is a composite of condom use and contraception variables. The condom use question asked: "Did you use a male or female condom the last time you had sexual relations? (1) Yes, male condom, (2) Yes, female condom, and (3) No". Responses were dichotomised yes/no $(0.47 \%$ used female condoms). The contraceptive use question asked: "Do (you/your husband/partner) currently use any method to avoid becoming pregnant? If so, what method do you currently use?" Responses were categorised as affirmative if the participant reported using non-barrier modern contraception (birth control pill, hormonal injection). Final outcome categories included: (1) condoms only, (2) modern contraceptive only, and (3) dual methods (condoms+another modern contraceptive).

Consistent condom use, our secondary outcome, was measured with the question: "In the last 12 months, you used a male condom during sexual relations: (1) Always, every time, (2) Sometimes, or (3) Never?" Among condom users, eligible responses included always (consistent) or sometimes (inconsistent).
Covariates included socio-demographics (age, education, race, religion, region, urban, household wealth), method at sexual debut, fertility (number of children, desire to have children) and relationship status. Household wealth was a composite measure of household ownership of assets (electricity, number of rooms), source and means of water supply, sanitation facilities, and type of flooring. ${ }^{46}{ }^{47}$ Responses to categorical variables were first dichotomised, Principal Component Analysis was conducted to identify one principal factor $(\alpha=0.78)$, and a standardised summative score was created.

Method use at sexual debut was measured with the questions, "The first time [you had sex], did you use a condom?" and "Without counting condoms, did you use another form of protection against pregnancy during the first time?" Responses were categorised: (1) no method, (2) contraceptive method only, and (3) condom use.

\section{Statistical analysis}

Data analysis began with sample descriptive statistics and bivariate distributions between covariates and dual protection outcomes. Chi-square $\left(\chi^{2}\right)$ statistics (categorical variables) and ANOVA F-statistics (continuous variables) were used to identify statistically significant differences. Then, weighted multinomial logistic regression models estimated the un-/adjusted relative risk ratios (RRRs) of exclusive modern contraception and dual method use (vs exclusive condom use) $(\mathrm{n}=6017)$. Finally, among condom users $(n=3961)$, weighted logistic regression models estimated the un-/adjusted odds ratios (AORs) for consistent condom use. Multinomial logistic regression and logistic regression are more robust to assumptions of multivariate normality, homoscedasticity (equal variance) than linear regression, ${ }^{48}$ and they do not assume a linear relationship between the dependent and independent variables. ${ }^{48}$ However, we checked for empty cells using cross-tabulations, multivariate outliers using MATA with the -bacon- command in STATA, and collinearity among interval independent variables using the -pwcorr- command in STATA. Household wealth was negatively correlated with number of children, however removing either variable did not change the results of our models in any significant way, therefore we included both variables. ${ }^{48}$

\section{RESULTS}

Table 1 describes the national sample of all sexually active women who use modern contraception. Women were on average 28 years old, had 9 years of formal education, and 1.1 children. Approximately half wanted more children, most were married or in a civil union, $24 \%$ were single, $50 \%$ were of Black race, $63 \%$ were Catholic, 47\% lived in Southeastern Brazil, and $86 \%$ were urban residents. Nearly half used condoms at sexual debut. 
Table 1 Weighted distributions of socio-demographics, method at sexual debut, fertility, and relationship status in sexually active women (aged 15-49 years) using a modern method of contraception, Brazil $2006(N=6017)$

\begin{tabular}{|c|c|c|}
\hline Characteristic & $n$ & $\%$ \\
\hline \multicolumn{3}{|l|}{ Socio-demographics } \\
\hline Age (years) [mean, (SD)] & 6017 & $28.3(0.26)$ \\
\hline $15-19$ & 873 & 15 \\
\hline $20-24$ & 1545 & 25 \\
\hline $25-29$ & 1308 & 21 \\
\hline $30-39$ & 1637 & 26 \\
\hline $40-49$ & 654 & 13 \\
\hline Education (years) [mean (SD)] & 6017 & $8.8(0.09)$ \\
\hline Primary school or less & 1350 & 19 \\
\hline Middle school & 1762 & 29 \\
\hline Some high school & 2118 & 37 \\
\hline High school or more & 787 & 15 \\
\hline \multicolumn{3}{|l|}{ Race } \\
\hline White & 2565 & 43 \\
\hline Black & 3137 & 51 \\
\hline Other & 315 & 6 \\
\hline \multicolumn{3}{|l|}{ Religion } \\
\hline None & 530 & 9 \\
\hline Catholic & 4008 & 63 \\
\hline Evangelical & 1172 & 22 \\
\hline Afro-religion or other & 307 & 6 \\
\hline \multicolumn{3}{|l|}{ Region } \\
\hline Southeast & 1327 & 47 \\
\hline North & 897 & 6 \\
\hline Northeast & 1031 & 22 \\
\hline South & 1608 & 18 \\
\hline Mid-West & 1154 & 7 \\
\hline Urban & 1575 & 86 \\
\hline Household wealth score & 6017 & $0.1(0.03)$ \\
\hline \multicolumn{3}{|l|}{ Method at sexual debut } \\
\hline No method & 2071 & 31 \\
\hline Contraceptive method only & 1216 & 21 \\
\hline Condom & 2730 & 48 \\
\hline \multicolumn{3}{|l|}{ Fertility } \\
\hline Number of children [mean (SD)] & 6017 & $1.1(0.03)$ \\
\hline \multicolumn{3}{|l|}{ Wants (more) children } \\
\hline Yes & 2899 & 49 \\
\hline No & 2947 & 48 \\
\hline Don't know & 171 & 3 \\
\hline \multicolumn{3}{|l|}{ Relationship status } \\
\hline Single & 1370 & 24 \\
\hline Separated & 601 & 8 \\
\hline Civil union & 1969 & 31 \\
\hline Married & 2077 & 37 \\
\hline
\end{tabular}

Ns are unweighted; means and percentages are weighted.

$\mathrm{SD}$, standard deviation.

Exclusive condom use at last sex was most common (40\%), followed by current use of modern contraception (33\%), and use of dual methods (27\%) (Table 2). Among women who used non-barrier contraception,
Table 2 Weighted percentage distribution of contraception, condom, and dual method use by factors in sexually active women (aged 15-49 years) using a modern method of contraception, Brazil $2006(N=6017) \dagger$

\begin{tabular}{llll}
\hline Characteristic & $\begin{array}{l}\text { Contraceptive } \\
\text { only }\end{array}$ & $\begin{array}{l}\text { Condom } \\
\text { only }\end{array}$ & $\begin{array}{l}\text { Dual } \\
\text { methods }\end{array}$ \\
& $33 \%$ & $40 \%$ & $27 \%$ \\
$100 \%$ & $(N=2056)$ & $(N=2321)$ & $(N=1640)$ \\
\hline
\end{tabular}

Contraception type

Oral contraceptive pill

$82(1724)$

81 (1356)

Long-acting hormonal

18 (332)

injectable

Consistent condom use

(past 12 months)

Socio-demographics

Age (years) [mean

$(S D)]^{\star \star *}$

$$
\text { 15-19 }
$$

20-24

25-29

30-39

40-49

Education (mean, SD) ${ }^{* * *}$

Primary school or less

Middle school

Some high school

High school or more

$29.6(0.29)$

$28.7(0.52) \quad 26.0(0.29)$

Race*

White

Black

Other

Religion**

None

Catholic

17 (159)

$\begin{array}{llll}51 & (456) \quad 32 & (258)\end{array}$

31 (466)

$34 \quad(558) \quad 35 \quad(521)$

38 (509)

$\begin{array}{llll}32 & (410) \quad 30 & (389)\end{array}$

38 (669)

$\begin{array}{llll}39 & \text { (596) } \quad 23 & \text { (372) }\end{array}$

$36 \quad(253)$

53 (301)

$11(100)$

$8.3(0.14)$

$8.8(0.15) \quad 9.2(0.12)$

44 (624)

$\begin{array}{llll}38 & (459) & 18 & (267)\end{array}$

34 (574)

$\begin{array}{llll}40 & (718) \quad 26 & (470)\end{array}$

28 (627)

$40 \quad(847) \quad 32 \quad(644)$

$\begin{array}{llll}42 & \text { (297) } & 30 & \text { (259) }\end{array}$

\section{Evangelical}

28 (231)

Afro-religion or other

34 (954)

$37 \quad(870)$

29 (741)

32 (1018)

42 (1322) $25 \quad$ (797)

$43 \quad(129) \quad 35 \quad(102)$

Region***

Southeast

22 (84)

29 (150)

$40 \quad(216) \quad 31 \quad(164)$

37 (1471) 28 (1097)

$46 \quad(515) \quad 22 \quad(274)$

32 (383)

45 (119) $34 \quad(105)$

North

29 (453)

$41 \quad(484) \quad 30 \quad(390)$

$57 \quad(512) \quad 20 \quad(171)$

Northeast

23 (214)

34 (330)

South

Mid-West

42 (669)

$44 \quad(460) \quad 22 \quad(241)$

$\begin{array}{llll}27 & (433) \quad 31 & (506)\end{array}$

Urban $^{* * *}$

\begin{tabular}{|c|c|c|c|}
\hline No & $42(670)$ & $33(513)$ & $25 \quad(392)$ \\
\hline Yes & $31(1386)$ & $41(1808)$ & $28(1248)$ \\
\hline Household wealth score** & $0.0(0.04)$ & $0.1(0.04)$ & $0.2(0.04)$ \\
\hline \multicolumn{4}{|l|}{ Method at sexual debut *** } \\
\hline No method & 39 (815) & $40 \quad(805)$ & 21 (451) \\
\hline $\begin{array}{l}\text { Contraceptive method } \\
\text { only }\end{array}$ & $41 \quad(549)$ & $36 \quad(385)$ & $23 \quad(282)$ \\
\hline Condom & $25 \quad(692)$ & $41(1131)$ & 34 (907) \\
\hline \multicolumn{4}{|l|}{ Fertility } \\
\hline Number of children*** & $1.4(0.04)$ & $1.0(0.04)$ & $1.0(0.05)$ \\
\hline \multicolumn{4}{|l|}{ Wants (more) children*** } \\
\hline Yes & $27 \quad(783)$ & $41(1217)$ & 31 (899) \\
\hline No & $38(1205)$ & $38(1043)$ & $24(699)$ \\
\hline
\end{tabular}

Continued 
Table 2 Continued

\begin{tabular}{|c|c|c|c|}
\hline \multirow{3}{*}{$\begin{array}{l}\text { Characteristic } \\
\frac{100 \%}{\text { Don't know }}\end{array}$} & \multirow{2}{*}{$\begin{array}{l}\text { Contraceptive } \\
\text { only } \\
33 \% \\
(N=2056)\end{array}$} & \multirow{2}{*}{$\begin{array}{l}\text { Condom } \\
\text { only } \\
40 \% \\
(N=2321)\end{array}$} & \multirow{2}{*}{$\begin{array}{l}\text { Dual } \\
\text { methods } \\
27 \% \\
(N=1640)\end{array}$} \\
\hline & & & \\
\hline & $36 \quad(68)$ & $43 \quad(61)$ & $21 \quad(42)$ \\
\hline \multicolumn{4}{|c|}{ Relationship status*** } \\
\hline Single & 11 (153) & 52 (738) & 37 (479) \\
\hline Separated & $18 \quad(89)$ & $52(308)$ & 31 (204) \\
\hline Civil union & $42 \quad(854)$ & 32 (618) & $26 \quad(497)$ \\
\hline Married & $42 \quad(960)$ & $36 \quad(657)$ & $22(460)$ \\
\hline
\end{tabular}

$81-82 \%$ used the oral contraceptive pill whereas 18 $19 \%$ used a long-acting hormonal injectable. Among women who used condoms, $61 \%$ of exclusive condom users and $27 \%$ of dual method users reported past year consistent condom use.

Dual methods users were, on average, younger and had more formal education than exclusive condoms users (Table 2). Also noteworthy, women who used condoms during sexual debut, had less children, and who were single or separated, most frequently reported current use of exclusive condoms or dual methods. In weighted, adjusted multinomial logistic regression models (Table 3), women had a significantly greater adjusted RRR of dual method use (vs exclusive condom use) if they had some high school education $(\mathrm{RRR}=1.69)$, lived in the South $(R R R=1.59)$, and had children $(R R R=1.22)$, whereas women had a significantly lower adjusted $R R R$ of dual method use (vs exclusive condom use) if they were older $(40-49$ years; $R R R=0.18)$, lived in the North $(R R R=0.40)$ or Northeast $(R R R=0.61)$, and had children $(R R R=0.88)$, compared to their respective reference groups.

In weighted, adjusted binomial logistic regression models among women who used condoms (Table 4), women had a significantly greater $A O R$ of consistent condom use (vs inconsistent condom use) if they used condoms at sexual debut $(\mathrm{AOR}=1.84)$, did not want children $(\mathrm{AOR}=1.86)$, and were single $(\mathrm{AOR}=2.77) /$ separated $(\mathrm{AOR}=2.45)$, whereas women had a significantly lower $A O R$ of consistent condom use if they used dual methods $(\mathrm{AOR}=0.19)$, compared to their respective reference groups.

\section{DISCUSSION}

Two-thirds of women who use modern contraception from the Brazilian national sample reported dual protection (40\% exclusive condoms, $27 \%$ dual methods). Sixty-one per cent of exclusive condom users and $27 \%$ of dual method users reported past year consistent condom use. Dual method use (vs exclusive condom use) was associated with having some high school education, living in the South, and having more children. Consistent condom use was associated with condom use at sexual debut, desire to control fertility, single/separated relationship status, and exclusive condom use (vs dual method use). We situate these findings within the context of HIV prevention and family planning in Brazil and recommend targeted syndemic services.

Our finding that dual method use (vs exclusive condom use) correlates with having more children, links to women's need to avoid unintended pregnancy. This finding supports the hypothesis that condoms are viewed as less effective contraception. Non-barrier contraception is adopted to prioritise better fertility control once women have children, but adopting a non-barrier contraception to ensure fertility control may come at a detriment to protection against HIV. Dual method users also reported significantly less consistent condom use than exclusive condom users, a finding supported by other studies. ${ }^{31-33}$ More nuanced measures of fertility desires and relationship context can improve our understanding of how these factors interact with HIV risk perceptions over the course of a relationship.

Consistent condom use was associated with condom use at sexual debut, net of other factors like fertility desires and relationship status. Even though condoms are a male-controlled method and a considerable time lag may exist since sexual debut, extant research has also found a strong and consistent correlation between condom use at last sex and condom use at sexual debut among young people, despite gender. $^{49-51}$ Using nationally representative longitudinal data, condom use at sexual debut was found to increase the likelihood of condom use at most recent sex; a finding largely independent of stable demographic and personal characteristics and proximate attitudinal, behavioural and relationship factors. ${ }^{49}$ This study also corroborates findings from Brazilian data that demonstrate a strong relationship between condom use at sexual debut and condom use later in life. ${ }^{50} 51$

Although the lasting power of condom use at sexual debut warrants further investigation, this finding makes a compelling case to improve and expand Brazil's school-based HIV prevention programme which began in the mid-1990s to target youth. ${ }^{52}$ The school-based programme exists in only $60 \%$ of all Brazilian public schools and varies widely in implementation. ${ }^{52}$ Condom distribution in schools is also limited by local parental advisory boards, the Catholic Church, and conservative groups. ${ }^{52}$ Moreover, the school-based programme covers $30 \%$ of all elementary schools and $96 \%$ of all high schools, but many youth most at risk of acquiring HIV either do not attend, or stop attending, school before they are exposed to HIV prevention programmes. ${ }^{6}$ Programmes must also target youth who drop out of school and are unstably housed. ${ }^{6}$ 


\section{Research}

Table 3 Weighted multinomial logistic regression of variables on exclusive modern contraception and dual method use (vs condom use) in sexually active women of reproductive age (15-49 years) using a modern method of contraception, in Brazil, Pesquisa Nacional Demografica e da Saúde da Criança e da Mulher $2006(n=6017) \dagger$

\begin{tabular}{|c|c|c|c|c|}
\hline \multirow[b]{2}{*}{ Characteristic } & \multicolumn{2}{|c|}{ Unadjusted RRR (95\% CI) } & \multicolumn{2}{|c|}{ Adjusted RRR (95\% Cl) } \\
\hline & Contraception only & Dual methods & Contraception only & Dual methods \\
\hline \multicolumn{5}{|l|}{ Socio-demographics } \\
\hline \multicolumn{5}{|l|}{ Age (years) } \\
\hline $15-19$ & Ref. & Ref. & Ref. & Ref. \\
\hline $20-24$ & $2.71 * * *(1.75-4.19)$ & $1.63^{*}(1.08-2.45)$ & $1.94^{* *}(1.26-3.01)$ & $1.40(0.92-2.12)$ \\
\hline $25-29$ & $3.54^{* * *}(2.37-5.29)$ & $1.46(0.98-2.19)$ & $2.19^{* * *}(1.43-3.36)$ & $1.18(0.77-1.79)$ \\
\hline $30-39$ & $2.98^{* * *}(1.96-4.52)$ & $0.94(0.61-1.44)$ & $1.41(0.88-2.27)$ & $0.65(0.40-1.07)$ \\
\hline $40-49$ & $2.08^{* *}(1.22-3.56)$ & $0.34^{* * *}(0.20-0.58)$ & $0.74(0.41-1.31)$ & $0.18^{* * *}(0.10-0.34)$ \\
\hline \multicolumn{5}{|l|}{ Education } \\
\hline Primary school or less & Ref. & Ref. & Ref. & Ref. \\
\hline Middle school & $0.75(0.54-1.03)$ & $1.38(0.96-1.99)$ & $1.00(0.70-1.42)$ & $1.27(0.88-1.83)$ \\
\hline Some high school & $0.61^{*}(0.42-0.91)$ & $1.68^{*}(1.11-2.54)$ & $1.00(0.63-1.58)$ & $1.69^{*}(1.08-2.64)$ \\
\hline High school or more & $0.60 * *(0.40-0.88)$ & $1.50 *(1.01-2.23)$ & $1.14(0.68-1.91)$ & $1.51(0.91-2.49)$ \\
\hline \multicolumn{5}{|l|}{ Race } \\
\hline White & Ref. & Ref. & Ref. & Ref. \\
\hline Black & $0.81(0.63-1.04)$ & $0.75^{*}(0.59-0.96)$ & $0.87(0.66-1.15)$ & $0.87(0.66-1.13)$ \\
\hline Other & $0.54^{*}(0.32-0.92)$ & $1.01(0.58-1.73)$ & $0.71(0.38-1.30)$ & $1.07(0.62-1.84)$ \\
\hline \multicolumn{5}{|l|}{ Religion } \\
\hline None & Ref. & Ref. & Ref. & Ref. \\
\hline Catholic & $1.30(0.88-1.90)$ & $0.98(0.65$ to 1.46$)$ & $1.20(0.76-1.89)$ & $1.13(0.75-1.69)$ \\
\hline Evangelical & $0.99(0.63-1.56)$ & $0.64(0.39$ to 1.04$)$ & $0.80(0.48-1.32)$ & $0.67(0.41-1.09)$ \\
\hline Afro-religion or other & $0.63(0.34-1.18)$ & $0.99(0.56$ to 1.74$)$ & $0.69(0.34-1.40)$ & $1.09(0.60-1.98)$ \\
\hline \multicolumn{5}{|l|}{ Region } \\
\hline Southeast & Ref. & Ref. & Ref. & Ref. \\
\hline North & $0.57^{* * *}(0.43-0.76)$ & $0.47^{* * *}(0.34-0.65)$ & $0.43^{* * *}(0.31-0.60)$ & $0.40 * * *(0.29-0.56)$ \\
\hline Northeast & $1.09(0.83-1.43)$ & $0.68^{*}(0.49-0.94)$ & $0.79(0.60-1.05)$ & $0.61 * *(0.44-0.86)$ \\
\hline South & $2.20 * * *(1.70-2.84)$ & $1.59 * *(1.17-2.17)$ & $1.89^{* * *}(1.42-2.52)$ & $1.59 * *(1.16-2.16)$ \\
\hline Mid-West & $1.32^{*}(1.01-1.72)$ & $0.98(0.73-1.30)$ & $1.10(0.82-1.46)$ & $0.88(0.68-1.15)$ \\
\hline \multicolumn{5}{|l|}{ Urban } \\
\hline No & Ref. & Ref. & Ref. & Ref. \\
\hline Yes & $0.59^{* * *}(0.45-0.77)$ & $0.88(0.66-1.16)$ & $0.73^{*}(0.54-0.99)$ & $0.73(0.53-1.00)$ \\
\hline Household wealth & $0.90(0.81-1.00)$ & $1.09(0.97-1.23)$ & $0.98(0.83-1.14)$ & $1.07(0.91-1.27)$ \\
\hline \multicolumn{5}{|l|}{ Method at sexual debut } \\
\hline No method & Ref. & Ref. & Ref. & Ref. \\
\hline Contraceptive method only & $1.16(0.81-1.67)$ & $1.19(0.80-1.78)$ & $1.18(0.83-1.66)$ & $1.37(0.95-1.97)$ \\
\hline Condom & $0.63^{* * *}(0.50-0.79)$ & $1.54^{* *}(1.18-2.01)$ & $0.82(0.61-1.09)$ & $1.22(0.93-1.61)$ \\
\hline \multicolumn{5}{|l|}{ Fertility } \\
\hline Number of children & $1.33^{* * *}(1.21-1.47)$ & $0.95(0.84-1.06)$ & $1.14^{*}(1.03-1.27)$ & $1.22^{* *}(1.06-1.40)$ \\
\hline \multicolumn{5}{|l|}{ Wants (more) children } \\
\hline Yes & Ref. & Ref. & Ref. & Ref. \\
\hline No & $1.53^{* * *}(1.23-1.92)$ & $0.82(0.64-1.05)$ & $1.11(0.85-1.44)$ & $1.10(0.82-1.47)$ \\
\hline Don't know & $1.28(0.75-2.19)$ & $0.66(0.38-1.16)$ & $0.97(0.56-1.69)$ & $0.66(0.37-1.17)$ \\
\hline \multicolumn{5}{|l|}{ Relationship status } \\
\hline Single & $0.18^{* * *}(0.12-0.28)$ & $1.15(0.80-1.64)$ & $0.24^{* * *}(0.16-0.36)$ & $0.91(0.64-1.28)$ \\
\hline Separated & $0.29^{* * *}(0.19-0.44)$ & $0.96(0.66-1.40)$ & $0.30^{* * *}(0.20-0.47)$ & $0.99(0.67-1.46)$ \\
\hline Civil union & $1.12(0.86-1.47)$ & $1.33(0.99-1.79)$ & $1.15(0.88-1.50)$ & $1.23(0.92-1.64)$ \\
\hline Married & Ref. & Ref. & Ref. & Ref. \\
\hline
\end{tabular}

Adjusted model includes all variables.

${ }^{* * \star} p<0.001 ;{ }^{* *} p<0.01 ;{ }^{*} p<0.05$.

†Multinomial logistic regression reference category is 'Condoms'.

$\mathrm{Cl}$, confidence interval; $\mathrm{RRR}$, relative risk ratio. 
Table 4 Weighted binomial logistic regression of variables on consistent condom use among sexually active women of reproductive age (15-49 years) using condoms in Brazil, PNDS $2006(n=3961)$

\begin{tabular}{|c|c|c|}
\hline Characteristic & OR $(95 \% \mathrm{Cl})$ & AORt $(95 \% \mathrm{Cl})$ \\
\hline \multicolumn{3}{|l|}{ Method at sexual debut } \\
\hline No method & Ref. & Ref. \\
\hline Contraceptive method only & $108(0.80-1.47)$ & $1.12(0.80-1.57)$ \\
\hline Condom & $163^{* * *}(125-212)$ & $1.84^{* * *}(1.33-2.55)$ \\
\hline \multicolumn{3}{|l|}{ Fertility } \\
\hline Number of children & $086^{* *}(077-096)$ & $0.98(0.85-1.11)$ \\
\hline \multicolumn{3}{|l|}{ Wants (more) children } \\
\hline Yes & Ref. & Ref. \\
\hline No & $12(0.94-1.53)$ & $1.86^{* * *}(1.31-2.64)$ \\
\hline Don't know & $073(0.39-1.35)$ & $0.69(0.31-1.55)$ \\
\hline \multicolumn{3}{|l|}{ Relationship status } \\
\hline Single & $231^{* * *}(1.70-3.14)$ & $2.77^{* * *}(1.90-4.06)$ \\
\hline Separated & $210 * * *(1.44-3.04)$ & $2.45^{* * *}(1.57-3.83)$ \\
\hline Civil union & $069 * *(0.53-0.90)$ & $0.85(0.63-1.15)$ \\
\hline Married & Ref. & Ref. \\
\hline \multicolumn{3}{|l|}{ Dual method use } \\
\hline No & Ref. & Ref. \\
\hline Yes & $024^{* * *}(0.18-0.31)$ & $0.19 * * *(0.15-0.25)$ \\
\hline
\end{tabular}

Single/separated women were more likely than married women to use condoms (exclusively or as dual methods) and to use condoms consistently; a finding supported across diverse socio-cultural and epidemiological contexts. ${ }^{53}$ Although individuals are less likely to protect themselves within marriage, they may be at greater HIV/STI risk than expected because partner risk assessments in these contexts are often unknown or inaccurate, especially in the case of infidelity and drug use. ${ }^{55} 56$

In Brazil, as in many global contexts, there is a double standard around fidelity norms in marriage/ common-law unions and many accept that it is masculine/male nature to have several women. ${ }^{57} \mathrm{~A}$ study in São Paulo found that almost half of women had sexual relations with only one person in the past year, whereas the same was true for only $7 \%$ of men. ${ }^{58}$ The word for cheating in Brazilian Portuguese is a good reflection of this double standard. Whereas corno/cornudo is used to explain the disgrace and loss of honour brought to a man when cheated on, the word is rarely used to describe the contrary, insinuating that honour is not lost when a woman is cheated on. ${ }^{59}$ Even though Brazilian women admit to being worried that their husbands are unfaithful, they cite 'powerlessness' about the situation. ${ }^{58} \mathrm{~A}$ qualitative study revealed that women in committed relationships know they might be at risk, with one woman saying: "[there are] two kinds of women [who] run the greatest risk [of getting HIV]: the one who stays home and trusts her husband, and the one who turns tricks". 57

It is critical that HIV/STI prevention initiatives address this double standard, collect data on the proportion of HIV/STI transmission that occur in marital/ civil union relationships, and develop alternative HIV prevention strategies for married/in-union couples at high HIV/STI risk as condom use may not be realistic within these relationship contexts. Moreover, both men and women in Brazil dislike condom use in marital/civil union relationships because they interfere with sensation and mutual pleasure. ${ }^{57}$ Most women in Brazil report using condoms only when they begin a relationship, but not with steady partners, even when they know their partner is unfaithful. ${ }^{57}$ Negotiated safety is a potential risk reduction strategy for married/in-union couples at high HIV/STI risk in which an explicit agreement is made between sexual partners. ${ }^{60}$ This agreement includes getting HIV/STI tests together, communication around HIV/STI risk, and establishing condom use rules outside their relationship. ${ }^{61}$ Negotiated safety could also facilitate the integration of family planning and HIV counselling within the relationship context.

Findings from this study should be viewed in light of several limitations. Data are cross-sectional, which does not allow for causal inference, and are based on self-report, which may introduce measurement error from recall bias or social desirability. Additionally, questions regarding sexual activity and condom use in large surveys often involve limited depth and no contextualisation of behaviour. With more in-depth relationship and contraception/condom measures (length of relationship, trajectory of condom/other contraceptive use, and negotiation dynamics) we could better elucidate mechanisms that influence dual protection decisions. Furthermore, condom use questions require linkage to a relationship context as women may also have concurrent sexual partners. For example, researchers assume that married women report on condom use with their husbands, but inconsistent condom use may reflect consistent condom use with a casual partner and no condom use with their husband.

This study highlights correlates of dual method and consistent condom use among a Brazilian national sample of reproductive-aged women using modern contraception. Syndemic services may usefully target completed/large families (not wanting children), single/separated individuals and non-exclusive relationships. Our findings also support the expansion of school-based programmes to promote condom use at sexual debut and to integrate family planning education.

Funding UCLA Bixby Center on Population and Reproductive Health; UCLA Latin American Institute; UCLA Graduate Division; UCLA Center For AIDS Research (CFAR); National Institute on Drug Abuse (K01DA036439) (T32DA023356). 
Competing interests None declared.

Ethics approval UCLA.

Provenance and peer review Not commissioned; externally peer reviewed.

\section{REFERENCES}

1 UNAIDS. The Gap Report, Joint United Nations Programme on HIV/AIDS, 2014.

2 World Health Organization. Women's Health. Media Centre. 2013. http://www.who.int/mediacentre/factsheets/fs334/en/ [accessed 10 November 2014].

3 Royce RA, Seña A, Cates W Jr, et al. Sexual transmission of HIV. N Engl J Med 1997;336:1072-1078.

4 Sedgh G, Singh S, Hussain R. Intended and unintended pregnancies worldwide in 2012 and recent trends. Stud Fam Plann 2014;45:301-314.

5 MOS. Boletim Epidemiólogico AIDS/DST. Brasília: Ministério da Saúde do Brasil, 2014.

6 Bastos FI, Nunn A, Hacker MA, et al AIDS in Brazil: the challenge and the response. In: Celentano D, Beyrer C (eds), Public Health Aspects of HIV/AIDS in Low and Middle Income Countries. Springer, 2009:629-654.

7 Brasil. Pesquisa Nacional de Demografia e Saúde da Criança e da Mulher PNDS 2006; Relatório Final. Ministério da Saúde do Brasil. Brasília - D.F., Ministério da Saúde Centro Brasileiro de Análise e Planejamento.

8 Viellas EF, Domingues RM, Dias MA, et al. Assistência pré-natal no Brasil. Cadernos de Saúde Pública 2014;30: S85-S100.

9 Malarcher S, Olson L, Hearst N. Unintended pregnancy and pregnancy outcome: equity and social determinants. In: Blas E, Sivasankara K (eds), Equity, Social Determinants and Public Health Programmes. Geneva, Switzerland: World Health Organization, 2010:177-197.

10 Coelho EA, Andrade ML, Vitoriano LV, et al. Associaçao entre gravidez não planejada e o contexto socioeconômico de mulheres em área da Estratégia Saúde da Família. Acta Paul Enferm 2012;25:415-422.

11 Fusco CL, Silva Rde S, Andreoni S. Unsafe abortion: social determinants and health inequities in a vulnerable population in São Paulo, Brazil. Cad Saúde Pública 2012;28:709-719.

12 Prietsch SOM, González-Chica DA, Cesar JA, et al. Gravidez não planejada no extremo Sul do Brasil: Prevalência e fatores associados. Cad Saúde Pública 2011;27:1906-1916.

13 UNFPA). Planejamento Familiar No Brasil: 50 anos de história. Brasília: UNFPA, 2008.

14 Ferreira AL, Souza AI, Lima RA, et al. Research choices on contraceptive methods in post-abortion family planning clinic in the northeast Brazil. Reprod Health 2010;7:5.

15 Malta M, Todd CS, Stibich MA, et al. Patient-provider communication and reproductive health among HIV-positive women in Rio de Janeiro, Brazil. Patient Educ Couns 2010;81:476-482.

16 Diniz D. Abortion and public health in Brazil. Cad Saúde Pública 2007;23:1992-1993.

17 Diniz D, Medeiros M. Aborto no Brasil: uma pesquisa domiciliar com técnica de urna. Ciên Saúde Colet 2010;15 (Suppl. 1):959-966.

18 Diniz SG, d'Oliveira AF, Lansky S. Equity and women's health services for contraception, abortion and childbirth in Brazil. Reprod Health Matters 2012;20:94-101.
19 Victora CG, Aquino EM, do Carmo Leal M, et al. Maternal and child health in Brazil: progress and challenges. Lancet 2011;377:1863-1876.

20 Díaz M, Simmons R, Díaz J, et al. Expanding contraceptive choice: findings from Brazil. Stud Fam Plann 1999;30:1-16.

21 Prazeres F, Santiago L. Multimorbidity in primary care in Portugal (MM-PT): a cross-sectional three-phase observational study protocol. BMJ Open 2014;4:e004113.

$22 \mathrm{Ku} \mathrm{L}$, Sonenstein FL, Pleck JH. The dynamics of young men's condom use during and across relationships. Fam Plann Perspect 1994;26:246-251.

23 Singer M. Introduction to Syndemics: A Critical Systems Approach to Public and Community Health. Chichester, UK: Wiley, 2009.

24 Berer M. Dual protection: more needed than practised or understood. Reprod Health Matters 2006;14:162-170.

25 Crosby R, Salazar LF, DiClemente RJ, et al. Condom misuse among adjudicated girls: associations with laboratory-confirmed chlamydia and gonorrhea. J Pediatr Adolesc Gynecol 2007;20:339-343.

26 Trussell J. Contraceptive failure in the United States. Contraception 2011;83:397-404.

27 Bajos N, Warszawski J, Grémy I, et al. AIDS and contraception. Unanticipated effects of AIDS prevention campaigns. Eur J Public Health 2001;11:257-259.

28 Trussell J, Wynn L. Reducing unintended pregnancy in the United States. Contraception 2008;77:1-5.

29 Reynolds HW, Steiner M, Cates W. Contraception's proved potential to fight HIV. Sex Transm Infect 2005;81:184-185.

30 Hladik W, Stover J, Esiru G, et al. The contribution of family planning towards the prevention of vertical HIV transmission in Uganda. PLOS ONE 2009;4:e7691.

31 Cates W, Steiner MJ. Dual protection against unintended pregnancy and sexually transmitted infections: what is the best contraceptive approach? Sex Transm Dis 2002;29:168-174.

32 Tsuyuki K, Barbosa RM, Pinho AA. Dual protection and dual methods in women living with HIV: the Brazilian Context. J Sex Transm Dis 2013;2013:540789.

33 Peipert JF, Redding CA, Blume JD, et al. Tailored intervention to increase dual-contraceptive method use: a randomized trial to reduce unintended pregnancies and sexually transmitted infections. Am J Obstet Gynecol 2008;198:630.e1-630.e8.

34 Chibwesha CJ, Li MS, Matoba CK, et al. Modern contraceptive and dual method use among HIV-infected women in Lusaka, Zambia. Infect Dis Obstet Gynecol 2011;2011:261453.

35 Wilson TE, Koenig LJ, Walter E, et al. Dual contraceptive method use for pregnancy and disease prevention among HIV-infected and HIV-uninfected women: the importance of an event-level focus for promoting safer sexual behaviors. Sex Transm Dis 2003;30:809-812.

36 Sieving RE, Bearinger LH, Resnick MD, et al. Adolescent dual method use: relevant attitudes, normative beliefs and self-efficacy. J Adolesc Health 2007;40:275.e15-275.e22.

37 Crosby RA, DiClemente RJ, Wingood GM, et al. Correlates of using dual methods for sexually transmitted diseases and pregnancy prevention among high-risk African-American female teens. J Adolesc Health 2001;28:410-414.

38 Poppen PJ, Reisen CA. Women's use of dual methods of sexual self-protection. Women Health 2000;30:53-66.

39 MacPhail C, Pettifor A, Pascoe S, et al. Predictors of dual method use for pregnancy and HIV prevention among 
adolescent South African women. Contraception 2007;75:383-389.

40 Harvey SM, Henderson JT, Branch MR. Protecting against both pregnancy and disease: predictors of dual method use among a sample of women. Women Health 2004;39:25-43.

41 Juarez F, Legrand T. Factors influencing boys' age at first intercourse and condom use in the shantytowns of Recife, Brazil. Stud Fam Plann 2005;36:57-70.

42 Garcia S, Koyama MAH. Capitulo 6: Longevidade Sexual e Práticas Sexuais Desprotegidas: Desafios para a promoção da saúde sexual e reprodutiva das mulheres. Pesquisa Nacional de Demografia e Saúde da Criança e da Mulher (PNDS) 2006: Dimensões do Processo Reprodutivo e da Saúde da Criança. Ministério da Saúde do Brasil and Centro Brasileiro de Análise e Planejamento. Brasília, DF. (2009).

43 Brasil. Pesquisa Nacional de Demografia e Saúde da Criança e da Mulher PNDS 2006: Dimensões do Processo Reprodutivo e da Saúde da Criança. Ministério da Saúde, 2009.

44 IBGE. Pesquisa Nacional por Amostra de Domicílios: 2006. Rio de Janeiro: Instituto Brasilerio de Geografia e Estatística, 2007.

45 Blavatsky I. Avaliação e correção dos pesos de expansão de unidades pesquisadas na pesquisa PNDS 2006. Pesquisa Nacional de Demografia e Saúde da Criança e da Mulher PNDS 2006: Aspectos Metodológicos. Brasília, CEBRAP (Centro Brasileiro de Análise e Planejamento) and DECIT (Departamento de Ciência e Tecnologia/MS) (2008).

46 Filmer D, Pritchett LH. Estimating wealth effects without expenditure data - or tears: an application to educational enrollments in states of India*. Demography 2001;38:115-132.

47 Vyas S, Kumaranayake L. Constructing socio-economic status indices: how to use principal components analysis. Health Policy Plan 2006;21:459-468.

48 Hosmer DW Jr, Lemeshow S. Applied Logistic Regression. Chichester, UK: John Wiley \& Sons, 2004.

49 Shafii T, Stovel K, Davis R, et al. Is condom use habit forming?: condom use at sexual debut and subsequent condom use. Sex Transm Dis 2004;31:366-372.
50 Paiva V, Calazans G, Venturi G, et al. Age and condom use at first sexual intercourse of Brazilian adolescents. Rev Saúde Publica 2008;42(Suppl. 1):45-53.

51 Teixeira AM, Knauth DR, Fachel JM, et al. Adolescentes e uso de preservativos: as escolhas dos jovéns de três capitais brasileiras na iniciação e na última relação sexual. Cad Saúde Pública 2006;22:1385-1396.

52 Camargo K. Prevenções de HIV/AIDS: desafios mútiplos [HIV prevention: multiple challenges]. Divulgação Saúde Debate, 2003:70-80.

53 Anderson JE, Warner L, Macaluso M. Condom use among US adults at last sexual intercourse, 1996-2008: an update from national survey data. Sex Transm Dis 2011;38: 919-921.

54 De Walque D, Kline R. Variations in condom use by type of partner in 13 sub-Saharan African countries. Stud Fam Plann 2011;42:1-10.

55 Clark LF, Miller KS, Harrison JS, et al. The role of attraction in partner assessments and heterosexual risk for HIV. Understanding and Preventing HIV Risk Behavior: Safer Sex and Drug Use. Oskamp S \& Thompson SC. Thousand Oaks, CA, 2006, Sage: 80-99.

56 Ickovics J, Thayaparan B, Ethier K. Women and AIDS: a contextual analysis. In: Baum A, Revenson TA, Singer JE (eds), Handbook of Health Psychology. Mahwah, NJ: Lawrence Erlbaum Associates, 2001:817-839.

57 Hebling EM, Guimarães IRF. Mulheres e AIDS: relações de gênero e uso do condom com parceiro estável. Cad Saúde Pública 2004;20:1211-1218.

58 Goldstein DM. AIDS and women in Brazil: the emerging problem. Soc Sci Med 1994;39:919-929.

59 Parker RG. Bodies, Pleasures, and Passions: Sexual Culture in Contemporary Brazil. Vanderbilt University Press, 2009.

60 Corbett AM, Dickson-Gómez J, Hilario H, et al. A little thing called love: condom use in high risk primary heterosexual relationships. Perspect Sex Reprod Health 2009;41:218-224.

61 Calazans G, Araujo TW, Venturi G, et al. Factors associated with condom use among youth aged 15-24 years in Brazil in 2003. AIDS 2005;19(Suppl. 4):S42-S50.

\section{Major UK Sexual Health Charity to Close}

Sad news reached the journal as we went to press that the UK-based charity MEDFASH (Medical Foundation for HIV \& Sexual Health) will close at the end of 2016 due to dwindling funds. Founded in 1987 as an independent charity initially focused on AIDS, MEDFASH grew into other areas of sexual health, and developed a Europe-wide focus. It has sought to improve care for people with HIV and other sexual and reproductive health needs by offering information, guidance and support to providers, commissioners and policymakers. Often working in partnership, MEDFASH has reviewed implementation of national strategy, developed national and European guidance, developed service standards, reviewed services, examined the feasibility of national clinical audit, produced policy information bulletins, published educational materials and managed the delivery of training.

Outgoing Chief Executive Ruth Lowbury thanked all who have funded or collaborated with MEDFASH, including the British Medical Association, which first established and supported the charity. "There is still much to do to improve sexual health and the management of HIV, both in this country and beyond, and we hope those organisations will maintain their commitment and build on the achievements of MEDFASH", she said. MEDFASH is currently negotiating with other organisations to hand over its ongoing projects and publications. For further information contact: rlowbury@medfash.bma.org.uk; tel: 02073836345 or 07810181843 . 